\title{
Disminución de agudeza visual, hipertelorismo y exoftalmos debido a piomucocele bilateral
}

\section{Decrease in visual acute, hypertellorism, and exophthalms due to bilateral piomucocele}

$\mathrm{DOI}$

https://doi.org/10.35434/rcmhnaaa.2021.143.1279

\section{RESUMEN}

Introducción: El Piomucocele es la infección de un mucocele pre existente. Presentamos un varón de 22 años con disminución de agudeza visual, hipertelorismo y exolftamos. Presentó proptosis inferolateral y agudeza visual $20 / 80$ en el lado izquierdo. Sin antecedente de traumatismo, infecciones, pólipos o cirugías. TAC con imágenes sugestivas de mucocele bilateral con efecto compresivo orbital anterior en el lado derecho y posterior en el lado izquierdo. El efecto compresivo del piomucocele etmoidal y esfenoidal en el lado izquierdo produjo compromiso del canal óptico con disminución de la agudeza visual ipsilateral. Se realizó cirugía endoscópica nasosinusal con marsupialización y descompresión, encontrándose dos piomucoceles en dirección antero posterior bien delimitados en lado derecho y un piomucocele izquierdo. La evolución fue favorable. Ésta inusual presentación obliga a considerar el compromiso ocular insospechado, principalmente en piomucoceles que comprometan senos paranasales posteriores.

Palabras Clave: Hipertelorismo; Agudeza visual; Exoftalmia; mucocele (Fuente: DeCS-BIREME).

\section{ABSTRACT}

Background: The Piomucocele is the infection of a pre-existing mucocele. We present a 22-year-old man with decreased visual acuity, hypertelorism, and exophthalmos. He presented inferolateral proptosis and visual acuity $20 / 80$ on the left side. No history of trauma, infection, polyps, or surgery. CT scan with images suggestive of bilateral mucocele with anterior orbital compression effect on the right side and posterior on the left side. The compressive effect of the ethmoidal and sphenoid pyomukocele on the left side produced compromise of the optic canal with decreased ipsilateral visual acuity. Endoscopic sinonasal surgery was performed with marsupialization and decompression, finding two well-defined anteroposterior pyomukoceles on the right side and one left pyomukocele. The evolution was favorable. This unusual presentation forces us to consider unsuspected ocular involvement, mainly in pyomukoceles that involve posterior paranasal sinuses.

Keywords: Hypertelorism; Visual acuity; Exophthalmia; mucocele. (Source: DeCS-BIREME).
Esteban Vergara-de la Rosa ${ }^{1,2,3, a}$, Tomas GalvezOlortegui $^{4,5, a}$, Jose Galvez-Olortegui ${ }^{4,6, a}$

FILIACIÓN

1. Servicio de Otorrinolaringología, Hospital Regional Docente de Trujillo, Trujillo, Perú.

2. Facultad de Medicina, Universidad Nacional de Trujillo, Perú.

3. Unidad de Investigación Clínica, Scientia Clinical and Epidemiological Research Institute, Trujillo, Perú.

4. Unidad de Oftalmología Basada en Evidencias (Oftalmoevidencia), Scientia Clinical and Epidemiological Research Institute, Trujillo, Perú.

5. Departamento de Oftalmología, Hospital Nacional Guillermo Almenara Irigoyen, Lima, Perú.

6. Servicio de Oftalmología, Hospital Universitario Central de Asturias, Oviedo, España.

a. Médico Cirujano, Magíster en Investigación clínica.

\section{ORCID}

1. Esteban Vergara-de la Rosa / 0000-0002-7461-5775

2. Tomas Galvez-Olortegui / 0000-0002-2177-2849

3. Jose Galvez-Olortegui / 0000-0003-1818-9801

\section{CORRESPONDENCIA}

Esteban Vergara-de la Rosa

Scientia Clinical and Epidemiological Research Institute.

Mz. G Lt. 22 Urb. Vista

Hermosa -Trujillo - Perú

Teléfono: +51942957036

EMAIL

estebanvergara@scientiaceri.com

\section{CONFLICTOS DE INTERÉS}

Los autores niegan conflictos de interés.

\section{FINANCIAMIENTO}

Autofinanciamiento.

\section{REVISIÓN DE PARES}

Recibido: 05/05/2021

Aceptado: 20/09/2021

\section{COMO CITAR}

Vergara-de la Rosa, E., Galvez-Olortegui, T., \& GalvezOlortegui, J. Disminución de agudeza visual, hipertelorismo y exoftalmos debido a piomucocele bilateral. Revista Del Cuerpo Médico Hospital Nacional Almanzor Aguinaga Asenjo, 2021, 14(3), 390 - 393. https://doi.org/10.35434/rcmhnaaa.2021.143.1279

Esta obra está bajo una Licencia Creative Commons Atribución 4.0 Internacional. Versión Impresa: ISSN: 2225-5109 Versión Electrónica: ISSN: 2227-4731 Cross Ref. DOI: $10.35434 /$ rcmhnaaa OJS: https://cmhnaaa.org.pe/ojs 


\section{INTRODUCCIÓN}

El Piomucocele es la infección de un mucocele pre existente, siendo este último la lesión benigna, quística, expansible y de lento crecimiento más frecuente de los senos paranasales ${ }^{(1,2)}$; debido a su característica lítica y expansiva dentro de los senos paranasales son responsables de compromiso craneal y orbital.

Las teorías de la formación del mucocele y del piomucocele aún son inciertas, incluyen la degeneración quística de la glándula seromucosa, la erosión por presión intrasinusal y lo planteado por Lund ${ }^{(3)}$, quien propone que la obstrucción y la infección agregada estimulan la liberación de citoquinas que conducen a la producción de prostaglandinas y colagenasas, produciendo resorción ósea y generando la expansión sinusal ${ }^{(3)}$. Otros factores de riesgo incluyen infección respiratoria, traumas, cirugías y la enfermedad alérgica nasosinusal ${ }^{(4)}$.

Los mucoceles afectan en orden de frecuencia a los senos frontales, etmoidales, maxilares y esfenoidales ${ }^{(1,4-8)}$. Su presentación clínica depende del tamaño y de la dirección de su crecimiento hacia estructuras vecinas, por lo que generalmente existe un compromiso anatómico de órbitas y cráneo, que se acompaña de signos y síntomas variados como diplopía, cefalea, epifora, proptosis, disminución de la agudeza visual, obstrucción nasal e hipertelorismo ${ }^{(7-9)}$.

Su presentación unilateral es generalmente la más frecuente, siendo rara la presentación bilateral ${ }^{(2,7)}$. El compromiso bilateral generalmente es de tipo simétrico y compromete senos paranasales anteriores. Los mucoceles asimétricos, expansiones de los senos paranasales de ubicación anterior en un lado y senos paranasales de ubicación posterior en el lado contrario, son importantes debido a que podrían comprometer estructuras orbitales vitales de ubicación posterior como el nervio óptico. La presentación asimétrica es infrecuente, existiendo en la literatura científica consultada sólo 2 casos publicados, reportados como mucoceles, pero sin infección sobreagregada ${ }^{(2,7)}$. El diagnóstico del mucocele se sustenta en la evaluación clínica y el examen radiológico, siendo la tomografía axial computarizada el examen de elección ${ }^{(8)}$, aún en pacientes post operados por esta patología, por su alta recurrencia ${ }^{(7)}$.

Presentamos el caso de un paciente con piomucocele bilateral y asimétrico con compromiso principalmente del seno etmoidal anterior en el lado derecho, y del seno etmoidal posterior y esfenoidal en el lado izquierdo, produciendo proptosis, hipertelorismo y compromiso de la agudeza visual. Enfatizamos en la necesidad de considerar a la distribución asimétrica de los piomucoceles como un factor de sospecha diagnóstica de posibles complicaciones en la órbita.

\section{REPORTE DE CASO}

Paciente varón, de 22 años, atendido en el servicio de otorrinolaringología del Hospital Regional Docente de Trujillo, en la zona norte del Perú, por presentar hace 6 años una tumefacción a nivel de canto medial de ojo derecho de crecimiento progresivo, acompañado de epífora, cefalea frontal y obstrucción nasal progresiva ipsilateral. Hace 2 años se agrega tumefacción de canto medial de ojo izquierdo, complicada con obstrucción nasal bilateral, rinorrea transparente, aumento de epifora derecha y disminución de agudeza visual izquierda. Las molestias fueron de inicio espontáneo y curso progresivo, niega antecedentes de traumatismo, infecciones respiratorias, pólipos o cirugías previas.

La evaluación oftalmológica mostró proptosis bilateral a predominio del Ol, con desplazamiento de canto interno inferolateral, hipertelorismo (Figura 1), agudeza visual mejor corregida: OD 20/20 y OI 20/80, exoftalmometria: OD $21 \mathrm{~mm}$ Ol $24 \mathrm{~mm}$, sin alteración pupilar ni de la visión de colores ni del campo visual confrontacional.

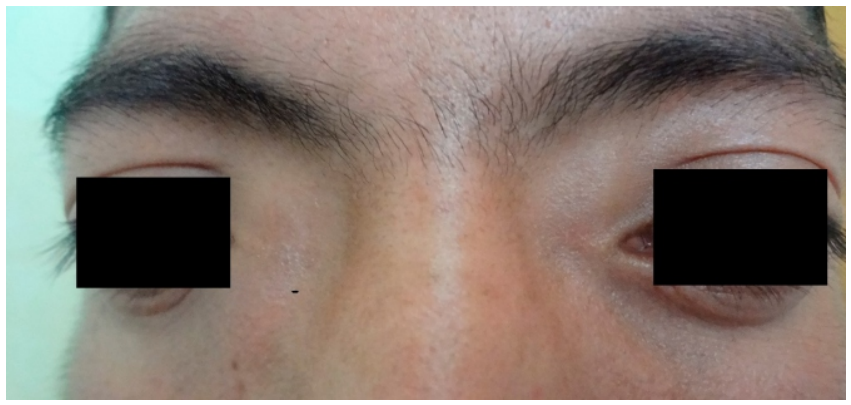

Figura 1.

Hipertelorismo y proptosis a predominio del ojo izquierdo.

Al examen endoscópico nasal, en la fosa derecha las estructuras intranasales formaban una masa blanda compuesta por cornete medio, bulla etmoidal y proceso uncinado adherida a septum nasal (figura 2). La fosa nasal izquierda presentó una masa blanda similar, pero de menor tamaño. Los exámenes sanguíneos de rutina estaban dentro de los valores normales.

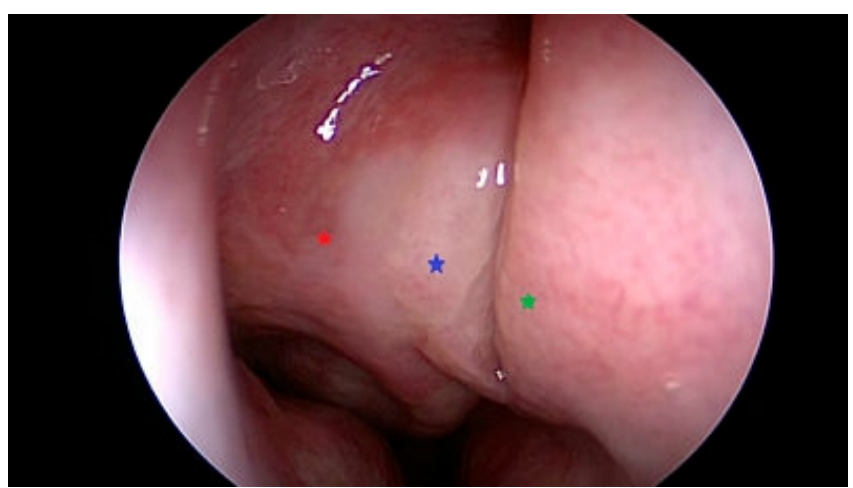

Figura 2.

Vista endoscópica de la cavidad nasal derecha. Desviación septal (estrella verde) adherida a una masa compuesta de cornete medio (estrella azul), bulla etmoidal y proceso uncinado (estrella Roja).

La tomografía de senos paranasales mostró imágenes de tejido blando bilateral, de apariencia homogénea, múltiple en cada lado y asimétricos, sugerentes de mucoceles. En el lado derecho los senos frontales, maxilares y principalmente el seno etmoidal anterior mostraron compresión a nivel de lámina papirácea, produciendo marcada lateralización del 
globo ocular. El mucocele del lado izquierdo comprometía los senos paranasales frontal y principalmente los senos etmoidal posterior y esfenoidal, ejerciendo una compresión posterior y lateral sobre el nervio óptico (Figura 3). No se evidenció compromiso intracraneal.

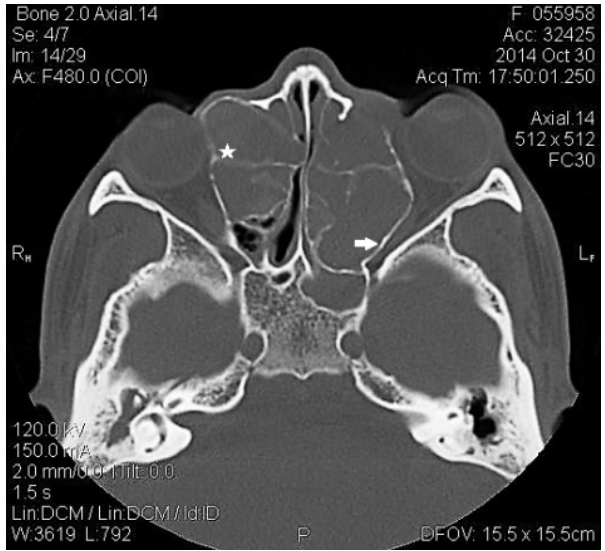

Figura 3.

TC axial que muestra proptosis y compresión orbital anterior derecha(estrella) y posterior izquierda, con compromiso del nervio óptico(flecha).

Se realizó marsupialización y descompresión, por vía endoscópica. Los puntos anatómicos referenciales estaban ausentes. En la fosa nasal derecha después de separar el septum nasal de una masa única compuesta por cornete medio, bulla etmoidal y proceso unciforme, se encontraron dos piomucoceles claramente separados en dirección antero posterior con secreción citrina y contenido purulento a la aspiración, separados por una pared ósea delgada bien delimitada (Figura 4). En la fosa nasal izquierda se identificó un piomucocele con menor cantidad de secreción purulenta. Ambas láminas papiráceas se encontraron erosionadas, con desplazamiento lateral debido a la presión externa ejercida en ambas órbitas. El reporte microbiológico del cultivo de la secreción purulenta fue informado como negativo para gérmenes comunes. El Paciente fue dado de alta al tercer día y fue controlado al décimo y trigésimo día post operatorio, donde se evidenció ausencia de epífora, sin cefalea ni obstrucción nasal, disminución de la proptosis y normalización de agudeza visual izquierda.

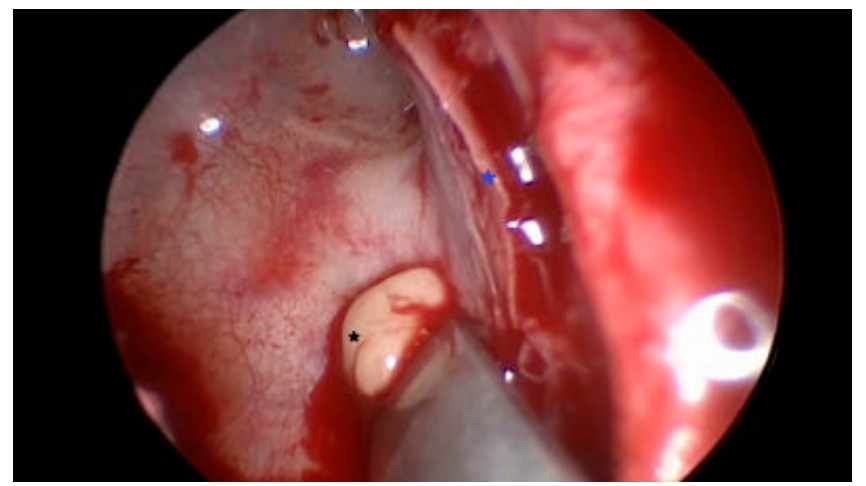

Figura 4.

Vista endoscópica intraoperatoria identificando dos piomucoceles en lado derecho. Se muestra la pared del primer piomucocele (asterisco azul) y secreción purulenta al abordar el segundo piomucocele en sentido antero posterior (asterisco negro).

\section{DISCUSIÓN}

Los piomucoceles son infrecuentes, aún más si son bilaterales y asimétricos. Varghese et al. reportaron en el 2004 el primer mucocele asimétrico que comprometía senos maxilares en un lado y etmoidales en el lado contrario ${ }^{(7)}$. Chong et al reportaron en el 2011 el segundo mucocele asimétrico con compromiso de los senos frontal y etmoidal en un lado y etmoidal en el otro $^{(2)}$. Reportamos un paciente con piomucocele bilateral y asimétrico.

La presentación asimétrica de los piomucoceles debe ser considerada como un factor importante de ayuda diagnóstica de posibles complicaciones, debido a que podría producir efectos compresivos en estructuras adyacentes en ambos lados de la nariz por efectos de expansión, remodelación y reabsorción ósea ${ }^{(1,3)}$. Dichos efectos, en nuestro caso, se presentaron a nivel anterior en lado derecho y posterior en el lado izquierdo, características no reportadas en otras publicaciones en pacientes con compromiso bilateral. En el presente caso, el efecto lítico y expansivo del piomucocele esfenoidal y etmoides posterior izquierdo originó compromiso del nervio óptico y disminución de agudeza visual; a diferencia del lado derecho, donde los efectos fueron de ubicación anterior, no presentando alteración de la visión.

La disminución de la agudeza visual izquierda fue debido, probablemente, al efecto compresivo y gradual del mucocele esfenoidal en el canal óptico, produciendo una inflamación secundaria, facilitado por el menor suministro vascular existente a dicho nivel ${ }^{(10)}$. La presencia de mucoceles, a pesar de su naturaleza benigna, justifica una evaluación oftalmológica que facilite información de las conductas médicas o quirúrgicas a seguir. En nuestro paciente, la intervención quirúrgica descompresiva facilitó la normalización de la agudeza visual a pesar del tiempo de enfermedad. Si bien la proptosis es uno de los signos más frecuentemente encontrados, debido al mayor compromiso de los senos frontales y etmoidales anteriores, la presencia del hipertelorismo también fue facilitado por el drenaje más complejo y variable de éstos senos y la compresión de la lámina papirácea, descrita como uno de los hallazgos tomográficos más frecuentes en los pacientes con mucoceles extensos $^{(11)}$.

El hallazgo de dos piomucoceles en el lado derecho se realizó en el intraoperatorio y no por imagen tomográfica, remarcando la necesidad de considerar el hallazgo intraoperatorio como un factor importante para el diagnóstico final de esta patología. La presencia de más de un piomucocele en el lado derecho de inicio espontáneo, aunado a la presencia de secreción purulenta con cultivo negativo, sugiere considerar la teoría propuesta por Lund et al., que reporta que los factores inflamatorios mediado por citoquinas (interleuquinas 1 y 6 ), prostaglandinas y moléculas de adhesión vascular, son los probables responsables de la destrucción ósea y no la obstrucción del seno, traumatismos o cirugías previas ${ }^{(3)}$, ausentes en el paciente.

El tratamiento de un piomucocele es eminentemente quirúrgico, y en nuestro caso fue realizado mediante cirugía 
nasosinusal, tratamiento actualmente recomendado debido a su menor morbilidad y adecuado abordaje. Considerando que los grupos etarios de mayor compromiso son la tercera y cuarta década ${ }^{(1,2,5)}$, la edad del paciente (22 años), obliga a seguir controles clínicos y radiológicos rigurosos debido a la alta recurrencia que presenta esta patología ${ }^{(12)}$. Finalmente, el limitado conocimiento de la etiología del piomucocele, plantea la necesidad de sospecha clínica de esta patología tanto en otorrinolaringología, oftalmología y cirugía de cabeza y cuello; con el objetivo de evitar posibles complicaciones en órbita y lograr, a través del abordaje multidisciplinario, un diagnóstico temprano.

\section{CONCLUSIÓN}

El piomucocele bilateral y de presentación asimétríco es raro. Esta presentación obliga a considerar el compromiso ocular insospechado, principalmente en piomucoceles ubicados en senos paranasales posteriores. Es importante su sospecha por otorrinolaringólogos, oftalmólogos y cirujanos de cabeza y cuello con el fin de tener diagnóstico y tratamiento precoz, evitando complicaciones.

\section{REFERENCIAS BIBLIOGRÁFICAS}

1. Arrué $P$, Kany $M T$, Serrano $E$, et al. Mucoceles of the paranasal sinuses: uncommon location. J Laryngol Otol. 1998;112(09):840-844.

2. Chong AW, Prepageran N, Rahmat O, Subrayan V, Jalaludin MA.
Bilateral asymmetrical mucoceles of the paranasal sinuses with unilateral orbital complications. Ear, Nose Throat J. 2011;90(2):2-5.

3. Lund VJ, Henderson B, Song Y. Involvement of cytokines and vascular adhesion receptors in the pathology of fronto-ethmoidal mucocoeles. Acta Otolaryngol. 1993;113(4):540-546.

4. Gulati R, Soni SK, Kumar A. Giant Ethmoid Mucocele Presenting as an Orbital Mass. Indian J Otolaryngol Head Neck Surg. 2015;67(1):97-99.

5. Kang IG, Kim ST, Jung JH, et al. Effect of endoscopic marsupialization of paranasal sinus mucoceles involving the orbit: a review of 27 cases. Eur Arch Otorhinolaryngol. 2014;271(2):293-297.

6. Sarac O, Bozdemir K, Yalciner G, Kutluhan A. Ocular manifestations of bilateral ethmoidal sinus mucopyocele: Case report. Turk Oftalmoloiji Derg. 2011;41(5):354-356.

7. Varghese L, John M, Kurien M. Bilateral asymmetric mucoceles of the paranasal sinuses: a first case report. Ear Nose Throat J. 2004;83(12):834-835.

8. Waizel-Haiat S, Díaz-Lara IM, Vargas-Aguayo AM, Santiago-Cordova $\mathrm{JL}$. Experience in the surgical treatment of paranasal sinus mucoceles in a university hospital. Cir Cir. 2016;85(1):4-11.

9. Williams V, lyer R, Reshma A, Jayashree M, Mohindra S, Verma S. Frontal pyomucocoele as forehead swelling in a five-year-old girl. Trop Doct. 2019;49(4):316-318. doi:10.1177/0049475519864249

10. Li E, Howard MA, Vining EM, Becker RD, Silbert J, Lesser RL. Visual prognosis in compressive optic neuropathy secondary to sphenoid sinus mucocele: Asystematic review. Orbit (London). 2018;37(4):280286

11. Lee TJ, Li SP, Fu CH, et al. Extensive paranasal sinus mucoceles: a 15year review of 82 cases. Am J Otolaryngol - Head Neck Med Surg. 2009;30(4):234-238.

12. Devars Du Mayne M, Moya-Plana A, Malinvaud D, Laccourreye O, Bonfils P. Sinus mucocele: Natural history and long-term recurrence rate. Eur Ann Otorhinolaryngol Head Neck Dis. 2012;129(3):125-130. 